\title{
Rosa 2000 utilisé avec succès à un quai sur pieux
}

\author{
Baguelin François $^{(\mathrm{a})}$, Sigros Bernard ${ }^{(\mathrm{b})}$, Derrien Pierre ${ }^{(\mathrm{b})}$, \\ Kovarik Jean-Bernard $^{(\mathrm{c})}$, Legros David ${ }^{(\mathrm{c})}$ \\ ${ }^{(a)}$ Fondaconcept, Pantin, France \\ ${ }^{(b)}$ E.M.C.C., Chevilly-Larue, France \\ ${ }^{(c)}$ Port Autonome de Rouen, France
}

\section{Résumé:}

Le Port Autonome de Rouen a expérimenté le format des Eurocodes sur la globalité d'un quai sur pieux (structure + fondations) en appliquant les recommandations Rosa 2000. Le retour d'expérience est présenté selon les points de vue des divers acteurs du projet. Le coût du quai n'est pas modifié par rapport à l'évaluation préalable basée sur un prédimensionnement «traditionnel». La méthode exige des vérifications dans des nombreuses situations de projet ce qui conduit à une multiplication des calculs. On peut attendre du format des Eurocodes d'apporter davantage de rigueur dans les hypothèses de calcul, d'améliorer la communication entre les acteurs du projet et de conduire à des ouvrages présentant un niveau de sécurité plus homogène et mieux explicité.

\section{Abstract:}

The Port of Rouen Authority has fully implemented the format of the Eurocodes to the design of Piling Berth (structure + foundations) based on the French Recommendations Rosa 2000. The lessons of experience are discussed according to the point of view of those who contributed to the design. The cost of the structure exhibits no significant difference in comparison to the preliminary approach based on a "traditional » design. The Eurocodes' method imposes a systematic check of numerous design situations, thus implying a demultiplication of calculations. One may expect from the Eurocodes to provide a more rigourous determination of the design conditions, to improve communication between the participants to the design procedure, and to get more homogeneous and explicit safety levels of the structures.

Mots-clés: Eurocodes, Semi-probabilisme, états-limites, ouvrage maritime, retour d’expérience.

Keywords: Eurocodes, semi probabilistic design, limit states design, maritime structure, lessons from experience.

\section{Introduction}

Les Eurocodes, normes européennes de justification des constructions fondées sur le format semi-probabiliste aux états-limites, voient progressivement le jour et ne tarderont pas à s'imposer aux concepteurs de bâtiments et d'ouvrages de génie civil. Cette méthode décrite dans la norme européenne ${ }^{(1)}$ référencée EN 1990 « Bases du calcul » a la maîtrise des risques pour objectif, ce qui ne signifie pas leur suppression totale, mais leur écrêtement à un niveau jugé acceptable. Elle conduit à un dimensionnement optimisé des ouvrages pour un niveau de sécurité homogène et explicite; les matériaux seront donc bien répartis, là où ils sont nécessaires, sans surdimensionnement. 
Ce format de vérification permet d'identifier plus clairement les incertitudes tout en recherchant constamment l'homogénéité des approches pour les diverses parties de l'ouvrage.

Mais, en abordant le cas des ouvrages en site aquatique, le concepteur est confronté à des problèmes spécifiques ${ }^{(2)}$ : les actions ne sont en général pas décrites par des textes réglementaires mais sont laissées à la « libre » appréciation du concepteur, qui dispose pour cela de peu de lignes directrices. Les textes de référence officiels restent incomplets, sont obsolètes ou peu compatibles.

Le Port Autonome de Rouen a choisi le projet de construction du quai sur pieux $\mathrm{n}^{\circ} 3$ d’Honfleur (désigné dans la suite par «QSH3 ») dans l'estuaire de la Seine ${ }^{(3)}$ pour expérimenter sur la totalité de l'ouvrage (structure et fondations) la mise en oeuvre du format des Eurocodes en se basant sur les recommandations applicatives générales Rosa 2000 publiées par le CETMEF (Centre d'Etudes Techniques Maritimes et Fluviales), l'autorité nationale compétente. Le cédérom Rosa 2000 fournit dans ce but au projeteur une aide hypertexte indispensable à la recherche et à la navigation dans cet ensemble très structuré de textes $^{(4)}$. Rosa 2000, entièrement cohérent avec le format de vérification des Eurocodes, propose des valeurs des coefficients partiels appropriés aux ouvrages en site aquatique et aux actions qui s'y exercent, de façon à permettre les raccordements indispensables. Il a développé en accord avec la norme européenne EN 1990 «Bases du calcul » les coefficients " de valeur » applicables à un paramètre donné du calcul et les coefficients " de modèle » déclinés par état-limite.

Il s’agit d'un quai à marchandises diverses, dont des trafics de bois importants, avec l'accueil de navires de croisière, de $137 \mathrm{~m}$ de long avec deux files de pieux dont l'une est intégrée dans un rideau mixte de soutènement des terres en arrière, lui-même ancré sur un contre-rideau par deux nappes de tirants inclinés. La plate-forme est en béton armé d'une conception optimisée par l'entreprise consistant en une succession de voûtes préfabriquées (figure 1). A cet endroit de l'estuaire, le marnage atteint 7 à $8 \mathrm{~m}$ en vives-eaux tandis que le courant de marée, héritier du fameux «mascaret ", porte à $2 \mathrm{~m} / \mathrm{s}$. Le quai accueillera des navires de mer de 60000 tonnes de déplacement.

On présente le retour d'expérience de cette démarche innovante et attendue par les constructeurs d'ouvrages en site aquatique, sur divers thèmes déclinés selon les points de vue du maître d'ouvrage (Port Autonome de Rouen), du maître d'œuvre (Direction de l'Aménagement et de l'Environnement - Service du Domaine et des Aménagements), du bureau de reconnaissances géotechniques (Fondasol), du bureau d'études de l'entreprise (E.M.C.C.) et du bureau de contrôle (Fondaconcept).

Une version plus développée de cet article a été présentée au colloque « Les risques et le génie civil » des 17-18 mars 2004 à Hammamet (Tunisie). 


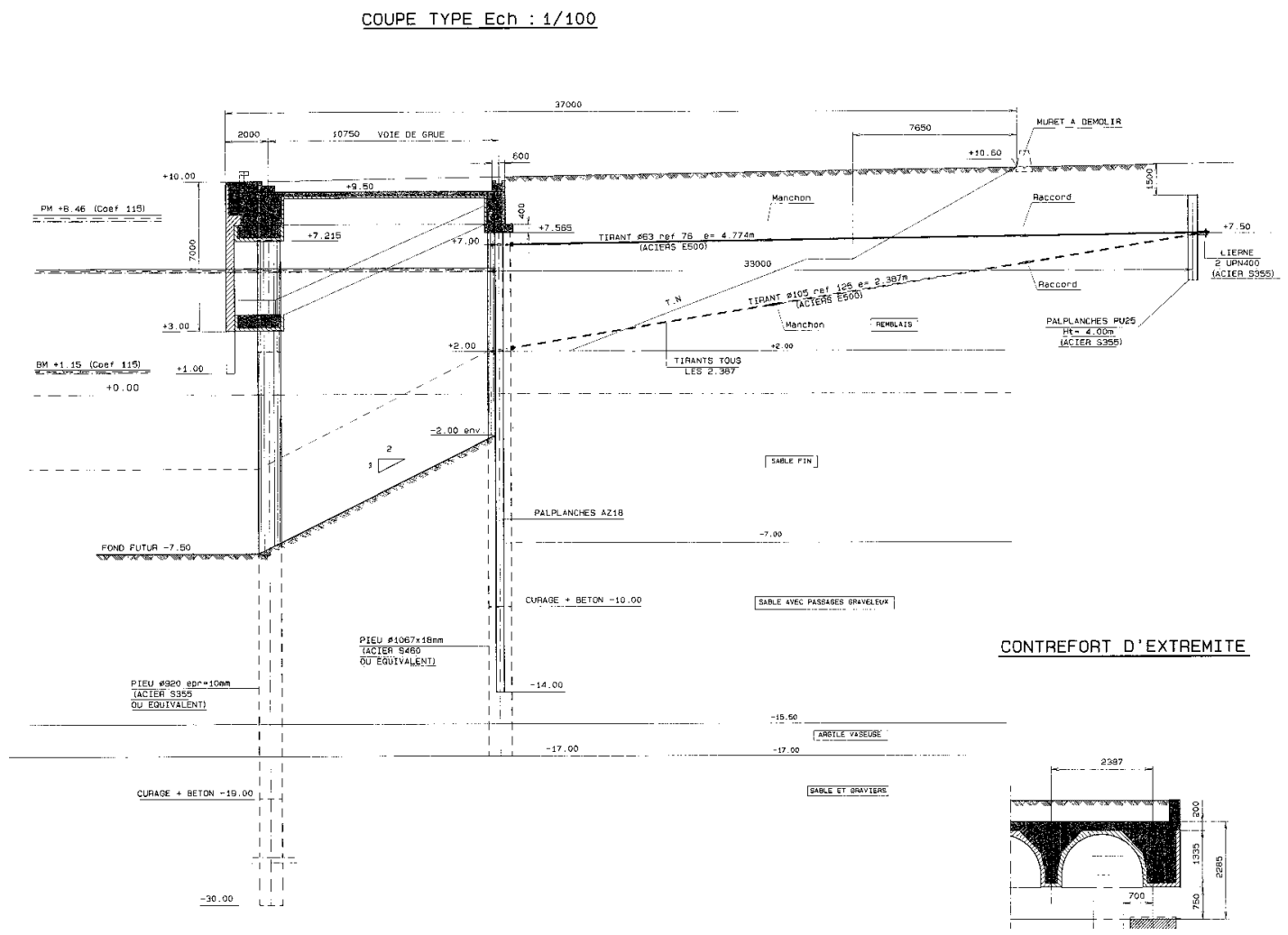

Figure 1. Coupe de travers de l'ouvrage (variante retenue)

\section{$\underline{\text { 2.Sur le programme de l'ouvrage et le cahier des charges contractuel }}$}

L'application des Eurocodes conduit le maître d'œuvre à suivre une démarche particulièrement rigoureuse pour la rédaction du cahier des charges et la détermination des hypothèses de calcul (figure 2). La démarche structurée du format de vérification requiert de déterminer avec la plus grande attention un nombre important de données, notamment ici :

- les valeurs représentatives des paramètres de sol,

- les valeurs représentatives des actions, en particulier les niveaux d'eau dans le terre-plein et en Seine, les charges d'exploitation sur le quai et l'effort d'accostage,

- les coefficients d'accompagnement à utiliser dans les combinaisons d'actions.

Jusqu'à ce que la méthode devienne d'usage courant, il est recommandé de tenir compte de la nouveauté du référentiel Eurocodes-Rosa 2000 par un allongement de la durée de la période de préparation (ici 2 semaines supplémentaires accordées par rapport aux « standards » du Port). 


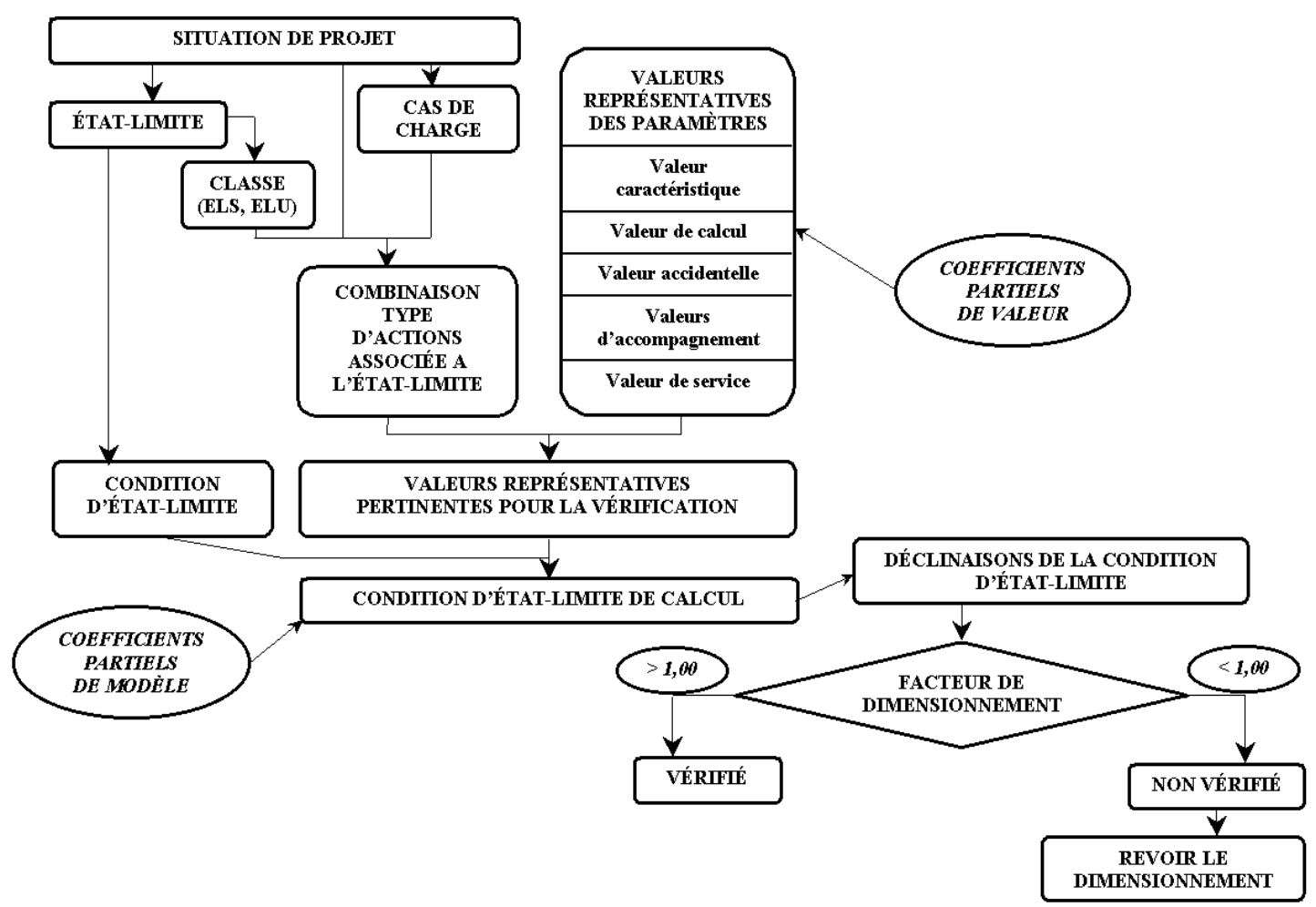

Figure 2. Logigramme des vérifications (extrait de Rosa 2000)

\section{Sur les aspects géotechniques}

\subsection{Une détermination soignée des "valeurs caractéristiques »}

La principale innovation de l'approche des Eurocodes pour le bureau d'études chargé de la reconnaissance préalable concerne les valeurs à fournir au bureau d'études " structures » pour les divers paramètres de sol. L'application des Eurocodes a de ce fait débuté dès l'amont du projet par le soin apporté à la détermination des valeurs caractéristiques des propriétés des sols faisant suite à la reconnaissance géotechnique. La détermination des valeurs représentatives était aisée dans le cas de ce quai car le terrain est homogène en plan. Dans la démarche applicative de Rosa 2000, il s’agit ${ }^{(5)}$ (figure 3) :

- en un premier temps, de donner pour chaque couche de sol et chaque paramètre $\mathrm{X}$ deux valeurs représentatives comme propriétés intrinsèques du sol, à savoir une valeur moyenne inférieure $\mathrm{X}_{\mathrm{mi}}$, et une valeur basse $\mathrm{X}_{\mathrm{b}}$ « au risque de $5 \%$ »;

- en un deuxième temps, de déterminer la valeur caractéristique $X_{k}$ pour chaque étatlimite, correspondant à un mécanisme de rupture particulier, ou plus généralement à un mécanisme d'interaction entre sol et structure. C'est l'étendue de la zone d'interaction qui commande la position de $\mathrm{X}_{\mathrm{k}}$ dans la fourchette $\left[\mathrm{X}_{\mathrm{mi}}, \mathrm{X}_{\mathrm{b}}\right]$;

- enfin on détermine la valeur de calcul $X_{d}$ par application du coefficient partiel $\gamma_{M}$ à la valeur caractéristique $X_{k}$. 


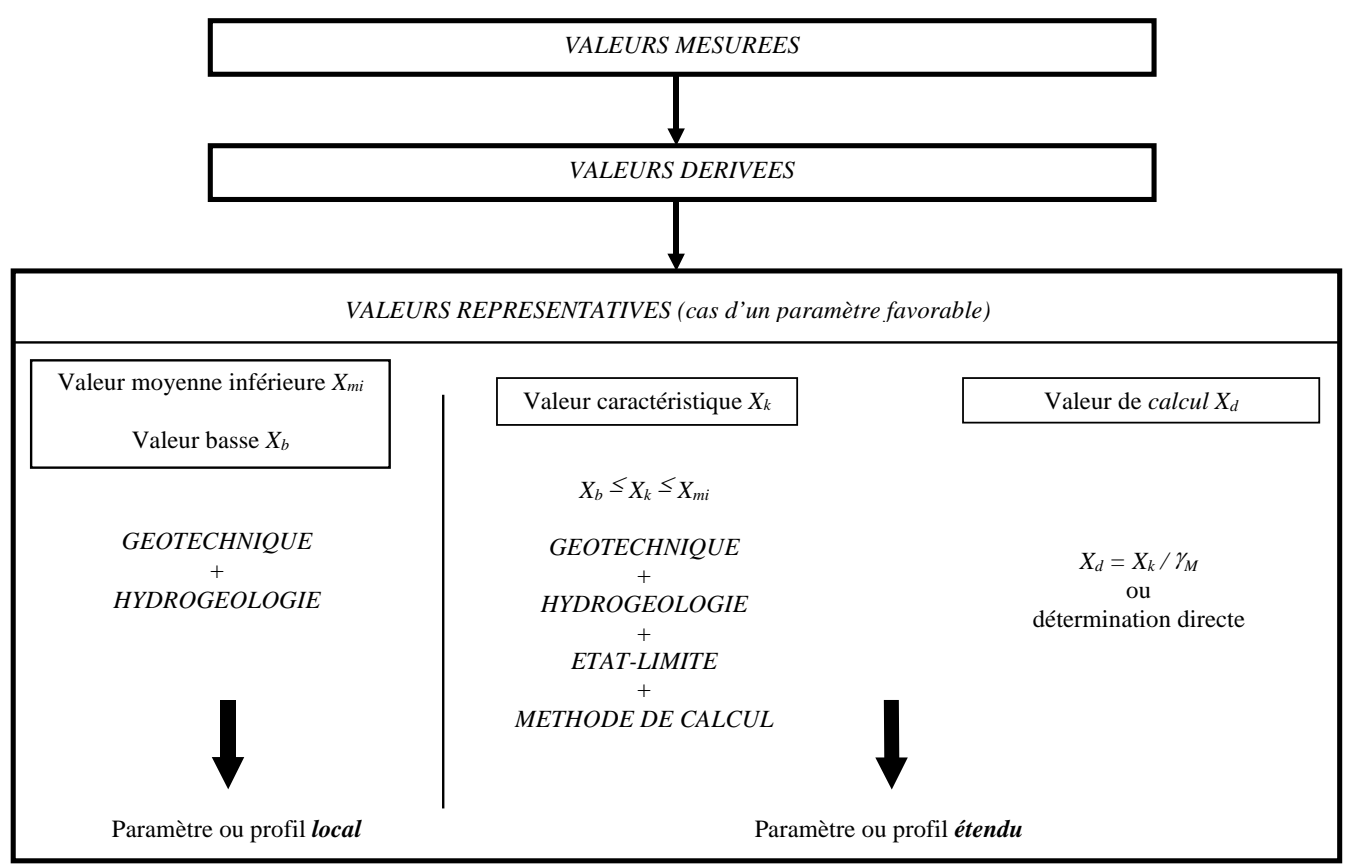

Figure 3. Les valeurs représentatives des propriétés des sols (extrait de Rosa 2000)

\subsection{Application au quai sur pieux}

On a retenu comme mécanisme d'interaction principal celui du rideau de soutènement de la deuxième file. La taille importante des prismes de poussée et de butée conduit à rapprocher sensiblement la valeur $\mathrm{X}_{\mathrm{k}}$ de la valeur moyenne mesurée. Il faut noter comme simplification le fait qu'on s'intéresse aux prismes de poussée et butée dans la phase d'interaction finale, où le dragage et le remblaiement sont maximaux.

Le long du fût du pieu (frottement latéral, réaction horizontale), il se produit un effet de réduction de la dispersion (variance) par « lissage » géométrique.

Le point principal est de bien choisir le modèle statistique : lorsque la dispersion est significative (coefficient de variation $\mathrm{CV}>25 \%$ ), et c'est le cas général pour $\mathrm{p}_{1}^{*}$ et encore plus pour $\mathrm{E}_{\mathrm{M}}$, il convient de fixer les valeurs représentatives à partir du modèle log-normal, plutôt que normal.

\subsection{Essai de comparaison avec l'approche « traditionnelle "}

A titre indicatif, l'angle de frottement des sables fins situés entre +3 CM (Cote Marine) et - $8 \mathrm{CM}$ a été évalué à $35^{\circ}$ dans l'approche traditionnelle, et à $39^{\circ}$ pour la valeur caractéristique. A noter que, pour la valeur de calcul, le coefficient partiel de sécurité sur $\operatorname{tg}(\varphi): \gamma_{\varphi}=1,20$, donne une valeur de calcul $\varphi_{\mathrm{d}}$ de $34^{\circ}$, finalement assez proche de la valeur traditionnelle.

Bien souvent dans les C.C.T.P., les valeurs présentées comme «valeurs caractéristiques » sont des «valeurs de calcul » car chaque intervenant (ici le géotechnicien) applique un « principe de précaution » à son niveau. Le bureau d'études de l'entreprise recommande que les valeurs des paramètres géotechniques du C.C.T.P, présentées comme «valeurs caractéristiques », soient plus optimistes que les «valeurs traditionnelles » pour que l'application des coefficients partiels ne conduise pas à dépasser les niveaux de dimensionnement raisonnables. Il apparaîtrait en effet trop pénalisant d'associer abruptement 
les valeurs «traditionnelles » des paramètres de sol et les coefficients de sécurité de l'approche semi-probabiliste.

\subsection{Le principe de cohérence}

Il a été retenu de ne pas pondérer les valeurs de c' et $\varphi$ ' à utiliser comme entrées du module de réaction dans la formule de Monnet, contrairement à l'application rigoureuse du principe de cohérence, à la fois parce que le produit final est un paramètre de déformation, usuellement considéré à sa valeur caractéristique dans les combinaisons fondamentales, et parce qu'ainsi on avait les mêmes valeurs de c', $K_{p}$ et $K_{o}$ dans les combinaisons rares et fondamentales, lesquelles s'enchaînaient dans certaines simulations

Rosa 2000 recommande de ne pas modifier les propriétés des sols au cours d'un même simulation des phases de chargement (méthodes au coefficient de réaction) mais de réitérer des simulations complètes avec des valeurs différentes des sols considérées ab initio. Les applications de méthodes d'éléments finis pourraient toutefois faire évoluer ce principe ${ }^{(6)}$ : les chargements resteraient appliqués avec un sol de propriétés constantes dans le " temps », prises égales aux valeurs caractéristiques; toutefois à chaque étape du chargement on simulerait l'effet de l'abaissement des propriétés du sol à la valeur de calcul pour vérifier que les états-limites ultimes ne sont pas atteints ; cet abaissement des propriétés géotechniques serait ensuite oublié et le chargement suivant serait appliqué à partir de l'état de contraintes/déformations précédent.

\section{Sur la complexité des Eurocodes}

L'élaboration automatique des combinaisons et de leur déclinaisons peut conduire à une perte de vigilance du projeteur vis-à-vis de la sélection des déclinaisons réellement pertinentes. La mise en forme des déclinaisons retenues pour le calcul doit donc être suivie de la démarche habituelle qui consiste à examiner de façon concrète et logique les possibilités qu'elles illustrent et à s'assurer, même de façon empirique, que ne manque pas «la » combinaison dimensionnante.

Est-il réellement pertinent de multiplier les modèles et les simulations alors même que l'évaluation des actions et leur concomitance est faite, pour ce type d'ouvrage, avec une certaine approximation? N'est on pas en train de s'imposer de fastidieux calculs au second ordre alors que le premier ordre est difficile à appréhender avec une bonne précision ?

On retiendra la complexité qui se manifeste dans la définition des charges d'exploitation et de leurs concomitances. Le maître d'œuvre est responsable de la description des opérations d'exploitation en lien avec l'exploitant dans le cahier des charges (ex : travail simultané d'une grue sur rails et d'une grue mobile...). La mise en forme des combinaisons d'actions et de leurs déclinaisons a été laissée à l'entreprise. Il semblait en effet difficile pour le maître d'œuvre, voire impossible, de préciser de façon exhaustive dans un cahier des charges tous les cas de figure (géométrique) potentiels, d'autant que les variantes étaient autorisées.

On peut conclure sur ce point que l'application des Eurocodes, même si elle tend à systématiser les combinaisons de calculs, n'exonère pas le projeteur d'un minimum de réflexion pour " extraire" des cas d'étude pertinents (mais qui s'en plaindrait ?). Le plus grand soin doit donc être apporté à la limitation du nombre des déclinaisons de calcul. Pour ne pas perdre le sens critique, il vaut mieux faire d'abord des calculs « à la main » et comprendre le fonctionnement de la structure.

La structure était complexe, avec des nombreux sujets d'interaction sol/structure (soutènement, portique). Il a fallu définir les sous-structures et poser des choix pour la modélisation des interactions : pieux, rideau et tirants, contre-rideau, tablier de béton, parement béton, bracons, poutre d'accostage, défenses et boucliers. Le choix des modèles de 
calcul est indépendant de l'application des coefficients partiels, mais plus le modèle est sophistiqué, plus la démultiplication des cas de calcul devient coûteuse. On a utilisé ici RIDO 4 (soutènement) et CGV (modèle de nœuds et barres sur sol élasto-plastique). La disponibilité d'un outil informatique permettant de traiter automatiquement l'ensemble requis des combinaisons d'actions et de propriétés des matériaux est appelée des vœux de tous ceux qui ont participé à ce travail.

\section{$\underline{5 . S u r ~ l e s ~ a p p r o c h e s ~ d e ́ r i v e ́ e s ~ e t ~ s i m p l i f i e ́ e s ~}$}

Pour tirer le plus grand profit de la méthode structurée des Eurocodes, il est proposé que le pré-dimensionnement :

- prenne en considération les situations de projet, notamment accidentelles, les cas de charge, les valeurs caractéristiques des paramètres, ces éléments étant tous décrits de façon absolument rigoureuse ;

- puis, s’il faut aller vite, effectue les calculs avec les coefficients globaux ou contraintes admissibles (par exemple « $2 / 3 \sigma_{\mathrm{e}}$ ») et la combinaison type unique «traditionnelle » : $\Sigma \mathrm{G}+$ $\Sigma \mathrm{Q}$.

La solution variante proposée par l'entreprise n’a pas été pré-dimensionnée en appliquant la démarche complète de vérification aux coefficients partiels, le format complet nécessitant un nombre trop important de calculs compte tenu des logiciels techniques actuellement disponibles.

Le nombre de combinaisons à calculer, avec leurs enchaînements, peut vite devenir rédhibitoire si l'on ne cherche pas à simplifier les déclinaisons dès le départ, non seulement les cas de charge, mais aussi les combinaisons des valeurs des paramètres de matériaux, $\mathrm{y}$ compris les situations de corrosion / non corrosion.

Un dégrossissage préalable sur un modèle de structure simplifié peur être utile pour guider ces choix. On recommande de mener une étude de sensibilité avec un seul cas de charge en faisant varier : corrodé / non corrodé, valeurs hautes / basses des sols; puis, après identification des cas dimensionnants, approfondir avec les déclinaisons appliquées rigoureusement sur un nombre de cas volontairement réduit par cette analyse préalable.

Ici le dégrossissage a été fait sur un modèle de structure simplifié, en particulier pour l'interaction entre le rideau principal et le contre-rideau. Il a permis de valider l'approche simplifiée consistant à calculer les rigidités en sections non corrodées et sur la base d'une combinaison de charges quasi-permanente. De même, la vérification de la stabilité d'ensemble (méthode de Kranz) s'est faite en sections non corrodées, une analyse ayant montré que c'était le cas le plus défavorable.

\section{$\underline{\text { 6.Sur les aspects financiers }}$}

L'application des Eurocodes avec les coefficients de Rosa 2000 au projet de construction du QSH3 (vue aérienne du chantier figure 5) n'a pas conduit à des modifications techniques ou des surcoûts financiers significatifs par rapport au dimensionnement antérieur des quais voisins à l'amont (QSH1 et QSH 2) effectué avec les errements traditionnels.

On observe ici que les lits de tirants sont dimensionnés par des combinaisons différentes : le lit supérieur est commandé par son état-limite ultime (ELU) de résistance en situation accidentelle corrodée avec la combinaison type accidentelle, le lit inférieur par son état-limite de service (ELS) de résistance (durabilité) en situation durable non corrodée avec la combinaison type rare.

Traditionnellement on calcule les efforts dans l'ouvrage en situation non corrodée et l'on vérifie la résistance en situation corrodée. Ici on a mené une comparaison des calculs des 
efforts selon les situations corrodées et non corrodées. On conclut que le double calcul n'est pas vain. Il s'est manifesté un écart de 3 à $4 \%$ pour les efforts dans les palplanches, de 1 à $12 \%$ pour le lit de tirant inférieur et davantage pour le lit de tirant supérieur. En tout cas le calcul traditionnel était insécuritaire !

On peut aussi noter les évolutions suivantes :

- augmentation de la longueur des tirants à cause de l'état-limite de stabilité du massif interne (méthode de Kranz). Le coefficient de modèle pour Kranz ( $\left.\gamma_{\mathrm{d} \text {, fond }}=1,50\right)$ est-il trop élevé ? Le coefficient global utilisé pour la stabilité globale par Bishop est de 1,50 tandis que celui de Kranz est supérieur, entre 1,50 et 2,00 : ceci autoriserait à maintenir les $\gamma_{\mathrm{d}}$ fond de 1,25 avec Bishop, et de 1,50 avec Kranz.

- fiche des pieux : la longueur a augmenté sous l'effet conjugué des conditions de sol (contrôle de battage et procédure d'arrêt de battage) et des hypothèses de concomitances des charges d'exploitation au-delà des simplifications de l'avant-projet ;

- béton armé : le niveau de dimensionnement est inchangé à l'exception du ferraillage des voiles bas de la poutre de quai qui sont considérablement renforcés à cause de l'action d'accostage accidentel (choc entre les défenses).

Il est malaisé de faire la part des choses entre l'effet des coefficients partiels issus du format des Eurocodes et la prise en compte de situations ou d'actions accidentelles qui n'était pas incluses dans le programme traditionnel des vérifications mais que l'analyse des conditions de sécurité et de durabilité a fait inscrire au cahier des charges.

Le calage des différents coefficients partiels recommandés dans Rosa 2000 s'est donc avéré satisfaisant en comparaison des méthodes de pré-dimensionnement traditionnelles.

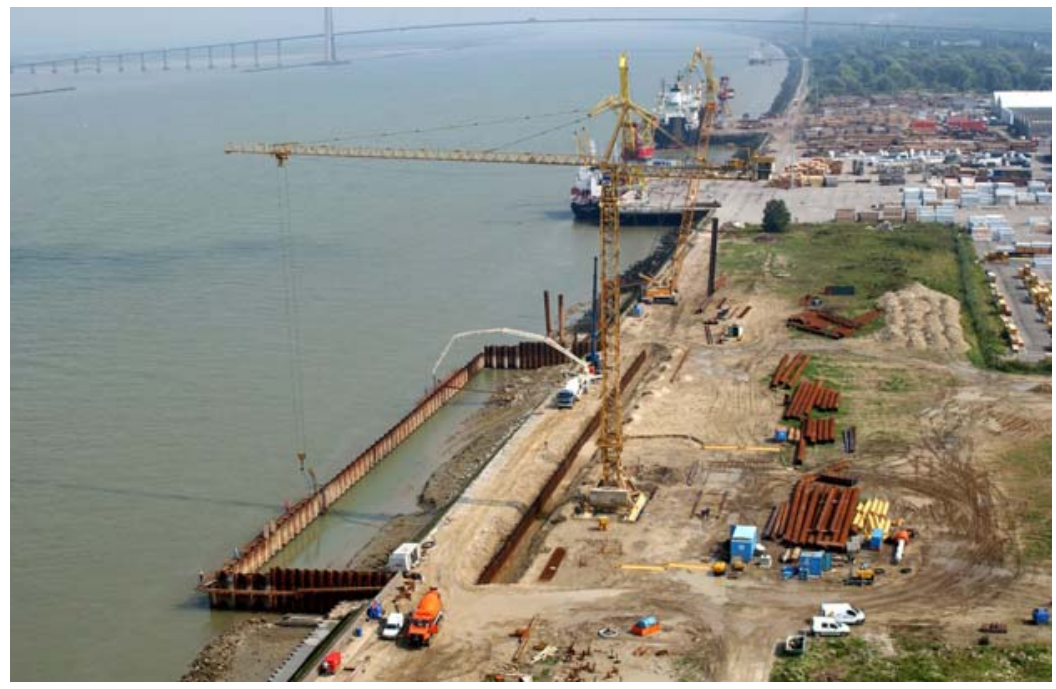

Figure 5. Vue aérienne du chantier - Bétonnage des pieux de la file 2

\section{Conclusion}

Le dimensionnement complet d'un ouvrage (structure et fondation) avec les Eurocodes est donc à la fois possible techniquement et raisonnable économiquement.

La démarche de vérification selon le format semi-probabiliste s'est révélé fructueuse par ce qu'elle apporte de rigueur notament à l'amont du projet dans la définition des hypothèses de calcul.

Les Eurocodes incitent à renforcer les reconnaissances préalables - sols, hydraulique - ce qui exige que chacun ait une bonne connaissance du référentiel, sous peine d'incohérence dans la fourniture des valeurs représentatives par exemple. 
La finalité «technique " des Eurocodes n'est pas de modifier l'économie des dimensionnements, elle est de permettre aux projeteurs d'y « voir plus clair » dans les divers coefficients appliqués et dans les approches de la sécurité, c'est-à-dire de traiter les incertitudes là où elles se trouvent dans une démarche rigoureuse et structurée qui améliore et homogénéise la fiabilité des constructions.

On peut attendre de la démarche «Eurocodes", après une phase de familiarisation indispensable et sous réserve d'une recherche commune de simplifications, d'apporter davantage de rigueur dans les hypothèses de calcul, d'améliorer la communication entre les acteurs du projet, de conduire à des ouvrages présentant des niveaux de sécurité plus homogènes et mieux explicités.

\section{Références}

1.Association française de normalisation (2003). Norme EN 1990, Bases de calcul.

2.Piet O., (2002). Le dimensionnement des ouvrages maritimes aux états-limites.La Houille Blanche $n^{\circ}$ 2-2002, 97-100.

3.Port Autonome de Rouen, (2002). Dossier de consultation des entreprises pour la construction du $3^{\text {ème }}$ quai en Seine à Honfleur.

4.Ministère de l'Equipement (France), (2001). Rosa 2000 - Recommandations pour le calcul aux états-limites des ouvrages en site aquatique.Centre d'Etudes Techniques Maritimes et Fluviales, Ponts-Formation-Editions.

5.Baguelin F., Kovarik J.-B., (2000). Une méthode de détermination des valeurs caractéristiques des paramètres géotechniques. Revue française de géotechnique $n^{\circ}$ 93, 35-41.

6.Simon B., (2003). Communication verbale lors du comité Rosa 2000 du 25 septembre 2003. 